\title{
Exercise performance and fatiguability in patients with chronic fatigue syndrome
}

\author{
Henry Gibson, Nadine Carroll, John E Clague, Richard H T Edwards
}

\begin{abstract}
To examine the role of delay in recovery of peripheral muscle function following exercise in the fatigue experienced by patients with the chronic fatigue syndrome (CFS) and to examine the influence of effort perception in limiting exercise performance in these patients, a study was carried out on a group of twelve patients with chronic fatigue syndrome and 12 sex and age-matched sedentary control subjects. Symptom limited incremental cycle exercise tests including measurements of perceived exertion were performed followed by examination of the contractile properties of the quadriceps muscle group for up to 48 hours. Muscle function was assessed by percutaneous electrical stimulation and maximum voluntary contractions. Muscle function at rest and during recovery was normal in CFS patients as assessed by maximum isometric voluntary contraction, 20:50 $\mathrm{Hz}$ tetanic force ratio and maximum relaxation rate. Exercise duration and the relationship between heart rate and work rate during exercise were similar in both groups. CFS patients had higher perceived exertion scores in relation to heart rate during exercise representing a reduced effort sensation threshold of 3.2 units on an unmodified Borg scale in CFS patients. Patients with chronic fatigue syndrome show normal muscle physiology before and after exercise. Raised perceived exertion scores during exercise suggest that central factors are limiting exercise capacity in these patients.
\end{abstract}

$(\Im$ Neurol Neurosurg Psychiatry 1993;56:993-998)

The chronic fatigue syndrome (CFS) comprises a constellation of symptoms with physical and mental fatigue as the predominant features. Consensus definitions have been published which both include the presence of fatigue for at least six months duration. ${ }^{12}$ Whilst aetiological factors producing this syndrome remain the subject of debate, patientsare considerably debilitated by the symptom of fatigue which is present at rest, made worse by exercise which is then followed by a prolonged period of exhaustion. ${ }^{34}$

In neurophysiological terms fatigue can be defined as failure to maintain a required or expected force. ${ }^{5}$ Using well-established physi- ological techniques muscle fatigue can be further categorised as "central" where there is volitional or non-volitional failure of neural drive to the muscles or "peripheral" where there is failure in force generation by mechanisms at or beyond the neuromuscular junction. Patients with CFS may, however, use the term "fatigue" in a different subjective sense of weariness or tiredness unrelated to objective measures of muscle fatigue. ${ }^{6}$ It is important therefore to establish whether the fatigue experienced by CFS patients is peripheral in origin, encompassing a persistant neurophysiological fatigue state within the muscle particularly following exercise, or is a consequence of an alteration in central drive. Exercise tolerance has been found to be reduced in CFS patients ${ }^{78}$ but motivational factors were not considered to be the primary cause. $^{8}$

To examine these factors this study has examined the response of a large proximal muscle group used in everyday activity, the quadriceps femoris, before and after exhaustive fatiguing dynamic activity in a group of patients with CFS. Changes in muscle characteristics were followed up to 48 hours to determine if delayed recovery occurred and if so whether it could explain the long-term excessive fatigue reported by CFS patients. Exercise capacity was also examined and central factors limiting exercise were studied using a Borg category scale to assess the perception of effort during exercise.

\section{Methods \\ Patients}

Twelve patients ( 6 men, 6 women) mean age 32.8 years (range $17-47$ years) attending the chronic fatigue clinic of one of the authors (RHTE) were studied. Subjects fulfilled the diagnostic criteria for the CFS determined at the meeting at Green College, Oxford, namely physical and mental fatigue for at least six months duration and present for more than $50 \%$ of the time. ${ }^{2}$ The mean duration of symptoms was 35.6 months (range: 10 months to 6 years). All subjects complained of excessive post-exercise fatigue. Seven subjects gave a history of a preceding viral illness before developing CFS. Three subjects were taking some exercise regularly, the other nine subjects were taking no regular exercise. A group of twelve age and sexmatched sedentary subjects were also studied as a comparison group, mean age 31.8 years (range 18-49 years). None of the control 
subjects took regular exercise. This study was approved by our hospital ethical committee and informed consent was obtained before the study from all subjects.

\section{Muscle function at rest}

Muscle function was assessed by dynanometry for both voluntary and stimulated contractions. ${ }^{9}$ The patients sat in a specially constructed chair with the knee flexed $90^{\circ}$ and isometric force was recorded via an inextensible strap placed around the ankle and attached to a force transducer. Two large pad electrodes $(12 \times 12 \mathrm{~cm})$ were strapped over the proximal and distal portions of the thigh for percutaneous stimulation. All subjects were encouraged verbally to make a maximal isometric voluntary contraction (MVC) of approximately three seconds duration, with and without twitch interpolation. ${ }^{1011}$ During twitch interpolation $1 \mathrm{~Hz}$ stimuli were superimposed on the voluntary contraction and the development of any additional twitch force signified submaximal effort. Verbal encouragement and visual feedback of performance, using an UV oscillograph (SE 3006/DL), was given throughout each contraction.

Physiological characterisation of muscle function to electrical stimulation was assessed by applying a train of impulses from a Devices 3072 stimulator driven by a computer (Apple IIe) over a frequency range of 1 , $10,20,50$ and $100 \mathrm{~Hz}$, each for 1 second (except that $10 \mathrm{~Hz}$ was given for 2 seconds to obtain a plateau force) as described previously. ${ }^{12}$ This permitted the frequency dependence of fatigue to be determined. The recorded force trace was termed the programmed stimulation myogram (PSM). Relaxation characteristics (MRR) were derived from the electronic differentiation of the force signal with respect to time at 100 $\mathrm{Hz}$ and both force and MRR were displayed on an UV oscillograph.

\section{Dynamic exercise testing}

An incremental exercise test was performed using a cycle ergometer (Monark 864) starting at $60 \mathrm{~W}$ and increasing by $30 \mathrm{~W}$ every three minutes until exhaustion. Heart rate was monitored at rest and during exercise. Subjects were also asked to rate perceived exertion (PE) using an unmodified Borg category scale every three minutes of the exercise test. ${ }^{13}$ The Borg scale is a well-established numerical grading system based on psychophysical principles for assessing perceived exertion. Subjects were familiarised with the scoring technique before the exercise study. Maximum heart rate was predicted from the formula 210 -(age $\times 0.65) .{ }^{14}$ Blood lactate levels were measured using an enzymatic technique $^{15}$ on samples taken via an indwelling IV cannula before, at the end and five minutes into the recovery period as a marker of anaerobic metabolism and exercise intensity. ${ }^{16}$

Muscle physiology after exercise

Muscle contractile properties were documented immediately following the exercise to monitor recovery of muscle function. Maximal voluntary contractions with and without twitch interpolation, and PSMs were performed during the acute recovery phase, at $5,15,30$ and 60 minutes after exercise and also during the late recovery period at 24 and 48 hours.

\section{Statistical analysis}

Maximum force generation in unfatigued muscle was compared to the predicted normal related to body weight ${ }^{9}$ calculated from the regression equation:

$$
\begin{aligned}
& \text { predicted maximum force }= \\
& 7 \cdot 91 \times \text { body wt }(\mathrm{kg})-37 \cdot 7 .
\end{aligned}
$$

Force generation was regarded as normal if the patient achieved a force within two standard deviations of predicted mean values.

Changes in maximal voluntary force following exercise was expressed as a percentage of initial resting values. Low frequency fatigue was assessed as change in the $20: 50 \mathrm{~Hz}$ force ratio and expressed as the absolute value.

Results are expressed as mean and 95\% confidence intervals unless otherwise stated as data were shown to be normally distributed using the Shapiro Wilk test. ${ }^{17}$ Statistical comparisons between groups were made using paired $t$-tests.

Pooled data from patients and controls were also analysed using the least squares method of linear regression analysis. ${ }^{17}$ The regression relationship between $\mathrm{PE}$ and heart rate was determined as an index of physiological strain ${ }^{18}$ and comparisons between patients and controls were made using analysis of covariance. The relationship between heart rate and work rate was also analysed using linear regression analysis of pooled data and comparisons between the groups were similarly made.

\section{Results}

All patients and controls completed the exercise and recovery study.

\section{Pre-exercise muscle physiology}

All patients and controls were able to produce maximum force. In five patients twitch interpolation indicated submaximal effort ( 1 man, 4 women) but all patients could achieve maximum force production with verbal encouragement. The PSM was similar for both patients and control groups and no significant differences in physiological characteristics were observed (table 1).

\section{Physiological response to exercise}

All subjects exercised to their subjective maximum exercise capacity. There was a wide variation in exercise time (table 2) in both patients and controls which were not significantly different between the groups. Both patient and control groups showed a similar linear increase in heart rate with work rate up to $120 \mathrm{~W}$, the absolute work rate over which all subjects could be compared (fig 1). 
Table 1 Physiological characteristics of quadriceps muscle function in CFS patients and controls

\begin{tabular}{lccl}
\hline & $\begin{array}{l}\text { Patients } \\
\text { mean }(95 \% \text { CI })\end{array}$ & $\begin{array}{l}\text { Controls } \\
\text { mean }(95 \% \text { CI })\end{array}$ & $p$ \\
\hline MVC (N) & $443(320-608)$ & $476(389-554)$ & $0 \cdot 431$ (NS) \\
20:50 Force Ratio & $77 \cdot 1(71 \cdot 8-81 \cdot 7)$ & $74.9(71 \cdot 4-79 \cdot 2)$ & 0.300 (NS) \\
MRR (100Hz) & $10.6(8 \cdot 3-11 \cdot 8)$ & $10 \cdot 4(8 \cdot 9-11 \cdot 4)$ & 0.601 (NS) \\
(\% force loss 10msec-1) & & & \\
\hline
\end{tabular}

Table 2 Resting, peak exercise and recovery physiological characteristics for CFS patients and controls

\begin{tabular}{|c|c|c|c|}
\hline & $\begin{array}{l}\text { Patients } \\
\text { mean }(95 \% \text { CI })\end{array}$ & $\begin{array}{l}\text { Controls } \\
\text { mean }(95 \% \mathrm{CI})\end{array}$ & $p$ \\
\hline \multirow{4}{*}{$\begin{array}{l}\text { Resting Heart Rate } \\
\left(\text { b min }{ }^{-1}\right) \\
\text { Resting PE } \\
\text { Peak Heart Rate } \\
(\text { b min-1) } \\
\text { Peak PE } \\
\text { Peak Work Rate } \\
\text { (Watts) } \\
\text { Exercise Duration } \\
\text { (mins) }\end{array}$} & $\begin{array}{l}81 \cdot 5(72-92) \\
7 \cdot 1(5 \cdot 9-8 \cdot 3)\end{array}$ & $\begin{array}{r}76 \cdot 8(66-84) \\
6 \cdot 2(5 \cdot 9-6 \cdot 4)\end{array}$ & $\begin{array}{l}0.314 \text { (NS) } \\
0.153 \text { (NS) }\end{array}$ \\
\hline & $\begin{array}{c}162 \cdot 7(144-178) \\
19 \cdot 2(18 \cdot 4-20)\end{array}$ & $\begin{array}{l}190(180-198) \\
19.1(18.5-19 \cdot 7)\end{array}$ & $\begin{array}{l}0.001 \\
0.809 \text { (NS) }\end{array}$ \\
\hline & $155 \cdot 0(120-210)$ & $175 \cdot 0(150-210)$ & 0.071 (NS) \\
\hline & $11 \cdot 7(8-16 \cdot 5)$ & $13.5(10.8-16.5)$ & 0.328 (NS) \\
\hline
\end{tabular}

Maximal predicted heart rate was achieved by all but one control subject suggesting that they were close or at their maximal working capacity but only two CFS patients achieved their target heart rate at exhaustion.

Resting blood lactate levels did not differ between the groups (1.93 (1.13 to 2.42) $\mathrm{mmol} / \mathrm{l} \mathrm{v} 1.82(0.93$ to 3.01$) \mathrm{mmol} / \mathrm{l}$ patients $v$ controls, $p>0.5$ ). At the end of exercise blood lactate levels were not different between the groups $(7.01$ (5.5 to $9 \cdot 1) \mathrm{mmol} / \mathrm{l}$ v $8.61(6.93$ to 9.15$) \mathrm{mmol} / \mathrm{l}$, patients v controls). At five minutes post exercise, when blood lactate levels are at near peak values ${ }^{19}$ there were significant differences between the groups $8.01(6.2$ to 10.1$) \mathrm{mmol} / 1$ v $10.43(9.0$ to 12.9$) \mathrm{mmol} / \mathrm{l}$, patients $\mathrm{v}$ controls, $\mathrm{p}<0.01$ ).

\section{Perception of effort during exercise}

There were no significant differences between the resting PE scores between the groups and PE scores were similar at the end of exercise. In patients $\mathrm{PE}$ scores increased linearly during exercise whereas in controls PE scores showed a threshold effect before sensation rose linearly. The regression analysis of pooled data of the linear portion of the PE:HR response showed that the increase in

Figure 1 Relationship between heart rate and work rate during incremental exercise for pooled data up to $120 \mathrm{~W}$ the work rate up to which all subjects could be compared. Similar linear increases in heart rate with work rate were seen in CFS patients (closed circles) and controls (open circles). Mean (SD) are shown for $n=12$ in each group.

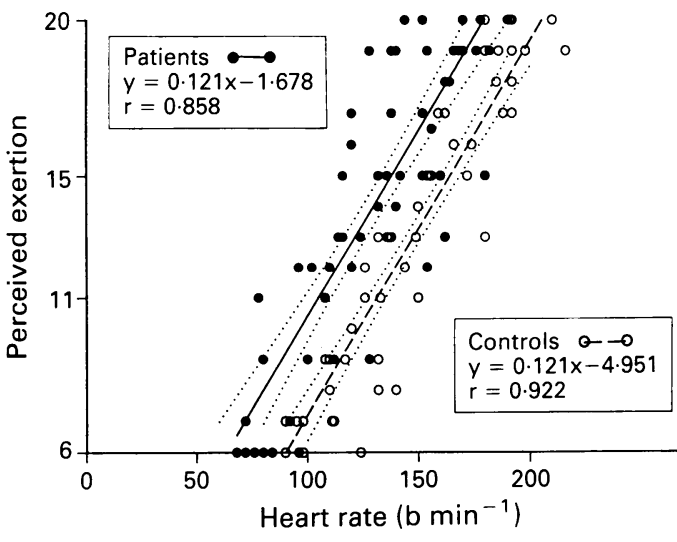

Figure 2 Relationship between perceived exertion and heart rate during incremental exercise. A parallel shift in slope to the left is seen for the patient group demonstrating a change in threshold for greater perception of effort compared with the control subjects. Data pooled, regression lines and $95 \%$ confidence intervals shown for $n=12$ in each group.

PE with HR during exercise did not differ between patients and controls (fig 2). However, the intercepts of these regression relationships were significantly different $(p<0.0001)$ representing the reduced threshold for sensation of $3 \cdot 2 \mathrm{PE}$ units in the patient group once exercise commenced.

\section{Muscle physiology after exercise}

Five minutes after exercise the MVC was reduced in both patients and controls compared with the pre-exercise value $(82 \cdot 2 \quad(70 \cdot 2$ to 90.2$) \%$ and $83.9(70.5$ to 97.2$) \%$ of preexercise values respectively). Full recovery of MVC to pre-exercise values was seen in both groups 24 hours post-exercise.

The mean $20: 50 \mathrm{~Hz}$ force ratio declined from $77 \cdot 1(71.8$ to $81 \cdot 7) \%$ and $74.9(71.4$ to $79 \cdot 2) \%$ before exercise to $57 \cdot 7 \quad(41.6$ to $75.5) \%$ and $49.4(41.9$ to 60$) \%$ at five minutes in patients and controls respectively $(\mathrm{p}=$ $0 \cdot 21$, NS). At 24 hours, recovery was almost complete in both groups (fig 3 ).

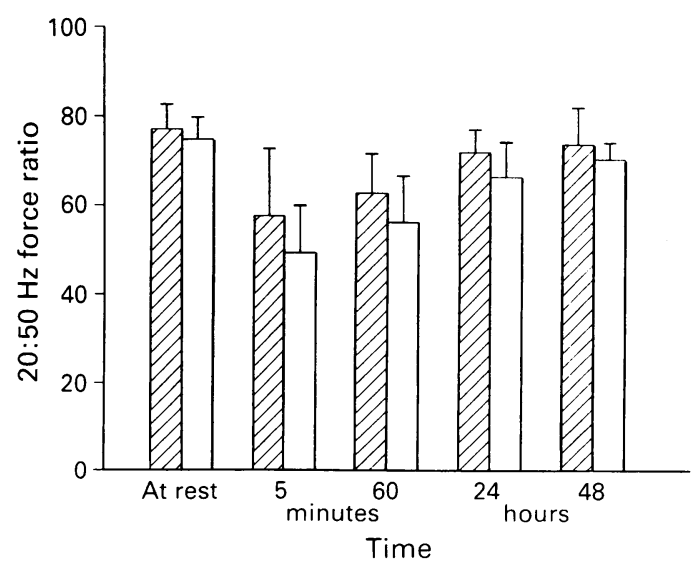

Figure 3 Acute and long-term recovery of 20:50 Hz force ratio following progressive cycle ergometry to exhaustion. No significant differences were observed between the patient (closed bars) and control (open bars) groups. Near complete recovery was attained by 24 hours after the exercise. Mean (SD) are shown, $n=12$ for each group. 
All 12 CFS subjects complained of the symptom of fatigue when retested at 24 and 48 hours. No control subject complained of fatigue. Eight CFS subjects complained of myalgia at retesting at 24 and 48 hours compared with three subjects in the control group.

Maximum relaxation rates (MRR) were not significantly different before exercise nor during recovery between patient and control groups. There were no significant changes in MRR measured five minutes after exercise from pre-exercise values in either group (table 2).

\section{Discussion}

We have studied the physiological function of a large muscle group before, during and after exercising activity in a well-defined population of patients with CFS. Our results support the previous findings that the contractile properties of skeletal muscle are normal. ${ }^{320} 21$ In addition we have shown that the long-term recovery of muscle is identical to normal subjects and complete by 24 hours in CFS patients following exhaustive exercise, even though patients characteristically complain of excessive fatigue, weakness or muscular discomfort at this time. Furthermore we observed differences in the perception of effort in relation to the physiological response to exercise indicating the likely involvement of central mechanisms in limiting exercise performance.

\section{Muscle physiology: at rest}

On first assessing MVC in the "fresh" state, several patients showed weakness of the quadriceps in relation to their body weight and had positive responses to twitch interpolation as seen by Stokes et al. ${ }^{20}$ This indicated that submaximal efforts were being produced, the stimulated twitch recruiting additional muscle fibres that were not being recruited by voluntary effort. It was found, however, that with the aid of visual feedback and verbal encouragement, maximal force generation could be achieved. Motivational or other central factors such as apprehension, possibly fear of pain, may be implicated in limiting force development, although in the present study no patient reported discomfort during the strength testing procedure. Other physiological factors that may influence force generating capacity such as reflex inhibition ${ }^{22}$ or inhibitory afferents arising in the muscles or joints ${ }^{23}$ may thus be excluded.

The analysis of the frequency dependence of muscle to electrical stimulation ${ }^{5}$ has led to the identification of 'high-' (loss of force at high stimulation frequencies) and 'low-' (loss of force at low stimulation frequencies) frequency fatigue. ${ }^{24}$ High frequency fatigue is short-lived and unlikely to occur during regular physical activity. Low-frequency fatigue, which is thought to arise from a failure in excitation-contraction coupling may last for a day or more following strenuous exercise. ${ }^{25}$ Persistence of this form of fatigue might explain the excess fatigue experienced by CFS patients. Abnormal EMG jitter has been described previously in post-viral fatigue patients $^{26}$ as have muscle biopsy abnormalities $^{27}{ }^{28}$ suggesting that abnormalities in muscle might contribute to symptoms in these patients. However, the histological changes reported are not consistent. ${ }^{29}$ Using the polymerase chain reaction to detect enteroviral RNA sequences, evidence of persistent viral infection has been reported in $53 \%$ of patients with post-viral fatigue and persistent viral infection has been postulated to interfere with cell metabolism and so cause fatigue. ${ }^{30}$ However, we have found no fatigue on electrophysiological examination in our patients. Muscle function is not impaired in these CFS patients and cannot be offered as an explanation of the apparent sensation of fatigue often experienced before exercise.

\section{Muscle physiology: recovery after exercise}

Few studies have followed characteristics of muscle function during recovery after activity and have not investigated long term delay of recovery. ${ }^{320}$ Persistence or delay in recovery of muscle function may contribute to the prolonged sensation of fatigue experienced by many patients with CFS following activity. The development of low-frequency fatigue is associated with the need for greater central "drive" to overcome the likely reduction in force development per impulse ${ }^{31}$ whereupon the resulting mismatch of afferent/efferent activity may further contribute to an alteration in sensation of effort. ${ }^{32}$ An analysis of the long-term recovery of muscle function in our study showed that while exhaustive dynamic exercise produced a significant and similar reduction in MVC and $20: 50 \mathrm{~Hz}$ force ratio compared to resting values in both groups, (the reduction of the latter reflecting the development of low frequency fatigue), recovery of MVC and 20:50 $\mathrm{Hz}$ force ratio were similar for both groups and complete by 24 hours. It is possible that the discomfort experienced by CFS patients may have arisen from the well documented post-exercise pain following unaccustomed exercise which usually peaks around 48 hours. ${ }^{33}$ This may have been prolonged in some patients in view of their reduced activity level before the exhaustive exercise test in this study.

\section{Exercise capacity}

There were wide differences in exercise capacity in both patients and controls as judged from exercise duration which may reflect the different degrees of fitness in the general population. Perceived exertion at the end of exercise did not differ between the groups but the finding of lower peak heart rate and reduced blood lactate levels five minutes after exercise suggests that the patient group were not exercising to their full capacity despite their high PE scores.

These data contrast with findings by other groups. Riley et al reported a trend towards higher heart rates at rest and a significantly greater heart rate compared with controls 
during submaximal treadmill exercise, but a non-significant difference at peak exercise. ${ }^{8}$ Montague et al found, as in the present study, a lower than predicted maximal heart rate in most CFS patients at the end point of exhaustive supine incremental cycle ergometry exercise and no difference in resting values compared with controls. ${ }^{7}$ Montague et al attributed the reduced end point heart rate in part to a slowing of acceleration of heart rate response after 12 minutes into exercise. However, as 20 of their 32 subjects were not able to exercise for this time (mean exercise duration $9 \cdot 1$ minutes) it is likely that only more fit CFS patients would continue exercise biasing heart rates towards lower values thus casting doubt on their conclusions. Moreover, comparison of heart rates between CFS patients and controls in our study for similar amounts of integrated work equivalent to 12 minutes cycling in the Montague study (810 and 750 Watts.mins respectively) showed a non-significant difference demonstrating no slowing of acceleration of heart rate. Riley et al attributed their findings to deconditioning, with oxygen uptake and lactate levels significantly lower in recovery. ${ }^{8}$ Our observations show an identical and linear heart rate/work rate response up to $120 \mathrm{~W}$, over which range all individuals exercised. We did not find the same degree of deconditioning in our CFS patients. The differences in the studies may reflect the differences in fitness in the general population. Patient symptoms in our study cannot be explained by deconditioning though it may contribute to exercise limitation in some CFS patients.

Of interest was the similar relative rise of PE with heart rate during exercise, but the marked different intercepts between the patient and control groups (fig 2). Perceived exertion has not been examined throughout exercise previously and although resting PE scores were similar in patients and controls, normal subjects did not perceive an increase in effort until after the second workload increment whereas patients had higher scores for perception of effort in relation to heart rate immediately on commencing exercise. In healthy subjects it is well established that leg effort during incremental cycle ergometry shows a threshold at 20 to 30 per cent of maximum power followed by a linear increase in effort rating. ${ }^{14}$ Thus it would appear that CFS patients have a lower threshold for sensation during exercise compared with the normal subjects. Heightened awareness of sensations from exercising muscle due to increased afferent activity has been suggested as a possible explanation of the symptoms experienced by these patients. ${ }^{20}$

Riley et al found higher perception scores in patients with CFS compared with their control groups but this difference was only recorded for peak exertion. ${ }^{8}$ Our controls and patients both reported maximum scoring of PE at exhaustion. Lloyd et al, who examined effort perception during submaximal muscle contractions of the elbow flexors in CFS patients found no subjective differences in submaximal effort perception. ${ }^{21}$ Riley et al suggested that the discrepancy in effort perception scoring may have resulted from a possible altered perception of their premorbid activity level, ${ }^{8}$ although it is surprising that at end exercise effort perception scoring was not maximal in both patients and controls. This may reflect the way in which the scoring was performed, that is, after the test rather than as the test ended as in our study. Lloyd et al found no difference in subjective measures of effort in both CFS patients and controls. ${ }^{21}$ These findings may reflect the nature of the activity performed since in that study a single muscle group was exercised submaximally whereas the cycling activity used in the present study involves the integration of several physiological systems and is more akin to "whole body" activity undertaken in everyday activity. The complex interaction of signals received by higher centres may compound the overall subjective sensation experienced during exercise.

Differences in effort perception scoring were observed immediately exercise started, at a time when activity was indeed submaximal. The involvement of metabolic factors contributing to a possible heightened afferent activity is possible but it is unlikely that electrophysiological factors such as impaired excitation-contraction coupling could have contributed so early on in the exercise. The increased PE scores during exercise may be due to CFS patients "adding on" the subjective fatigue felt at rest to their effort scores during exercise but this is speculative as no subjective measures of fatigue were made at rest and perceived resting effort was normal.

The finding of a reduced threshold for sensation in conjunction with the reduced peak heart rates and peak blood lactate levels (physiological indicators of submaximal exercise performance) suggest that central factors are likely to be contributing to the exercise limitation seen in these patients. These results in CFS patients are more in keeping with submaximal effort in exercise rather than solely lack of fitness or "deconditioning". It is possible that the sensory threshold during exercise and persistence of symptoms in CFS patients may be a learned response to stimuli no longer present, that is, the sensation threshold during exercise has been reset.

\section{Conclusions}

This study has shown that contractile properties of muscle in CFS patients are normal and patients show no excess fatiguability. Recovery of muscle function occurs identically to normal subjects. These CFS patients did not exercise to their full physiological capacity and exercise was accompanied by greater effort perception. A reduced sensation threshold was shown at the start of exercise representing the burden of their perceived "fatigue" at rest.

Graduated exercise programmes have been advocated as a means to improve activity and alleviate symptoms in CFS. ${ }^{34}$ The findings of 
this study should give confidence to patients that graduated exercise can be safely undertaken without risk of damage to their muscles. These findings support the need for further investigations to examine the ability of graduated exercise programmes or other treatments to reset the effort perception threshold in CFS patients.

Support from the Muscular Dystrophy Group of Great Britain and Northern Ireland and ICI Pharmaceuticals is gratefully acknowledged. We also thank Dr P MacLennan for performing measurement of lactate concentrations.

1 Holmes GP, Kaplan JE, Gantz NM, Komaroff AL Chronic Fatigue Syndrome: a working case definition. Ann Intern Med 1988;108:387-9.

2 Sharpe MC. A report-chronic fatigue syndrome: guidelines for research. F Roy Soc Med 1991;84:118-21.

3 Lloyd A, Hales J, Gandevia S. Muscle strength, endurance and recovery in the post infectious fatigue synance and recovery in the post infectious fatigue syn-

4 drome. F Neurol Neurosurg Psychiatry 1988;51:1316-22. Suppl 1986;711:179-88.

5 Edwards RHT. Human muscle function and fatigue. In: Porter R, Whelan J Eds. Human muscle fatigue: physiological mechanisms. London: Pitman Medical, 1981.

6 Wessely S, Edwards RHT. Chronic fatigue. In: Greenwood R, Banes M, McMillan T, Ward C, Eds. Neurological Rehabilitation. Edinburgh: Churchhill Livingstone, 1993:311-25.

7 Montague TR, Marrie TJ, Klassen GA, Bewick DJ, Horacek BM. Cardiac function at rest and with exercise in the chronic fatigue syndrome. Chest 1989;95:779-84.

8 Riley M, O'Brien CJ, Bell N, Nicholls DP, McCloskey DR. Aerobic work capacity in patients with chronic DR. Aerobic work capacity in patien

9 Edwards RHT, Young A, Hosking GP, Jones DA. Human skeletal muscle function: description of tests Human skeletal muscle function: description

10 Chapman SJ, Edwards RHT, Greig C, Rutherford O. Practical application of the twitch interpolation technique for the study of voluntary contraction of the quadriceps muscle in man. 7 Physiol (Lond) 1984;353:3.

11 Rutherford OM, Jones DA, Newham DJ. Clinical and experimental application of the percutaneous twitch superimposition technique for the study of human muscle weakness. I Neurol Neurosurg Psychiatry 1986;49: 1288-91.

12 Edwards RHT. Studies of muscular performance in normal and dystrophic subjects. $\mathrm{Br}$ Med Bull 1980;36: 159-64.

13 Borg G. Perceived exertion as an indicator of somatic stress. Scand F Rehab Med 1970;2:92-8.

14 Jones NL. Clinical exercise testing, 3rd ed. Philadelphia: Saunders WB, 1988:75-109.

15 Gutman I, Wahlefeld AW. In: Burgemeyer HV, ed.,
Methods of Enzymatic Analysis, 2nd ed. New York: Academic Press, 1974.

16 Shepard RJ. Physiology and Biochemistry of Exercise. New York; Praeger, 1985.

17 Armitage P, Berry G. Statistical methods in medical research. Oxford: Blackwell Scientific, 1987.

18 Edwards RHT, Melcher A, Hesser CM, Wigertz O, Ekelund L-G. Physiological correlates of perceived exertion in continuous and intermittent exercise with the same average power output. Eur $\mathcal{f}$ Clin Invest 1972; 2:108-14.

19 Astrand PO, Kaare R. Textbook of work physiology. Tokyo: McGraw-Hill, 1970:279-318.

20 Stokes MJ, Cooper RG, Edwards RHT. Normal muscle strength and fatiguability in patients with effort synstrength and fatiguability in patien
dromes. $B M \Im$ 1988;297:1014-17.

21 Lloyd AR, Gandevia SC, Hales JP. Muscle performance, voluntaby activation, twitch properties and perceived effort in normal subjects and patients with the chronic fatigue syndrome. Brain 1991;114:85-8.

22 Woods J, Furbish F, Bigland-Ritchie BR. Evidence for a fatigue-induced reflex inhibition of motoneuron firing rates. F Physiol (Lond) 1987;58:125-37.

23 Stokes MJ, Young A. The contribution of reflex inhibition to arthrogenous muscle weakness. Clin Sci 1984;67: 7-14.

24 Cooper RG, Edwards RHT, Gibson H, Stokes MJ Human muscle fatigue: frequency dependence of excitation and force generation. $\mathcal{F}$ Physiol (Lond) 1988;397: tion and

25 Edwards RHT, Hill DK, Jones DA, Merton PA. Fatigue of long duration in human skeletal muscle after exercise. of long duration in human skeletal

26 Jamal GA, Hansen S. Electrophysiological studies in the post-viral fatigue syndrome. $f$ Neurol Neurosurg Psychiatry 1985;48:691-94.

27 Behan PO, Behan WMH, Bell EJ. The postviral fatigue syndrome-an analysis of the findings in 50 cases. fInfect 1985;10:211-22.

28 Behan WMH, More IAR, Behan PO. Mitochondrial abnormalities in the postviral fatigue syndrome. Acta Neuropathol 1991;83:61-5.

29 Edwards RHT, Gibson H, Clague JE, Helliwell TR Muscle histopathology and physiology in the chronic fatigue Muscle histopathology and physiology in the chronic fatigue syndrome. In: Bock GR, Whelan J, eds. The chronic fatigue symd.

30 Gow JW, Behan WMH, Clements GB, Woodall C, Riding M, Behan PO, Enteroviral RNA sequences detected by the polymerase chain reaction in muscle of patients with postviral fatigue syndrome. $B M F 1991$ 302:692-6.

31 Newham DJ, Mills KR, Quigley BM, Edwards RHT. Pain and fatigue after concentric and eccentric muscle contractions. Clin Sci 1983;64:55-62.

32 Gandevia SC, McClosky DI. Changes in motor commands, as shown by changes in perceived heaviness, during partial curarization and peripheral a man. F Physiol (Lond) 1978;283:493-9.

33 Newham DJ. Plasma creatine kinase changes after eccentric and concentric contractions. Clin Sci 1986;64: 55-62.

34 Peel M. Rehabilitation in post viral syndrome. F Soc Occup Med 1988;38:44-5. 